\title{
Krawtchouk polynomials and quadratic semi-regular sequences
}

\author{
Stavros Kousidis \\ Federal Office for Information Security \\ Bonn, Germany \\ st.kousidis@googlemail.com
}

\begin{abstract}
We derive lower und upper bounds on the degree of regularity of an overdetermined, zero-dimensional and homogeneous quadratic semi-regular system of polynomial equations. The analysis is based on the interpretation of the associated Hilbert series as the truncation of the generating function of values of a certain family of orthogonal polynomials, the Krawtchouk polynomials.
\end{abstract}

\section{KEYWORDS}

Groebner bases; Semi-regular sequences; Degree of regularity; Hilbert regularity; Orthogonal polynomials; Krawtchouk polynomials

\section{INTRODUCTION}

Semi-regular sequences model generic homogeneous systems of polynomial equations as a generalization of regular sequences to the overdetermined case. They were designed to be algebraically independent, i.e. to have as few algebraic relations between them as possible, in order to assess the complexity of Faugère's Gröbner basis algorithm F5 [9]. The essential complexity parameter in that assessment is the degree of regularity, which is built in to the design of semi-regular sequences as a threshold up to which algebraic independence is maintained.

The degree of regularity of a semi-regular sequence essentially coincides with its Hilbert regularity, and can be computed by the power series expansion of a rational function and its truncation at the first non-positive coefficient. Asymptotic estimates of the degree of regularity via the analysis of this rational function by the saddle-point method of asymptotic analysis have been given by Bardet et al. in [3-6].

We follow a different approach to the degree of regularity in that we interpret the Hilbert series as the truncation of the generating function of values of a certain family of orthogonal polynomials, the Krawtchouk polynomials [17]. This will enable us to give various descriptions of the degree of regularity based on information about the location of extreme roots of the Krawtchouk polynomials. In particular, we will derive lower and upper bounds on the degree of regularity without any further restrictions on the systems we consider. That is, for any overdetermined, zero-dimensional and homogeneous quadratic semi-regular system $f_{1}, \ldots, f_{m} \in \mathbf{K}\left[X_{1}, \ldots, X_{n}\right]$ of polynomial equations with degree of regularity denoted by $d_{\text {reg }}$, we establish the lower bounds

$$
d_{\text {reg }} \geq \begin{cases}1+\left\lfloor\frac{1}{2}(2 m-n-2 \sqrt{m(m-n)})\right\rfloor & \text { Thm. 4.1, } \\ 1+\left\lfloor\frac{1}{2}\left(w_{4}^{6}-1\right)\right\rfloor & \text { Thm. 5.1, }\end{cases}
$$

where $w_{4}$ is the unique positive real root of the quartic polynomial

$$
q(w)=w^{4}-\frac{n}{\sqrt{2(2 m-n)}} w-6^{-\frac{1}{3}} i_{1} \quad\left(\text { with } i_{1} \approx 3.37213\right) .
$$

Furthermore, for such $f_{1}, \ldots, f_{m}$ we prove the upper bounds

$$
d_{\text {reg }} \leq \begin{cases}1+\left\lceil\frac{1}{2}\left(2 m-n+3-\sqrt{(2 m-n+1)^{2}-4 n^{2}}\right)\right\rceil & \text { Thm. 6.1, } \\ 1+\left\lceil x_{5}^{3}\right\rceil & \text { Thm. 7.1, }\end{cases}
$$

where $x_{5}$ is a particular positive real root of the sextic polynomial

$$
s(x)=x(x-1)^{2}\left(2 m-n-x^{3}\right)-\frac{1}{4} n^{2} .
$$

While the lower bounds are valid for any $m>n$, the existence of the upper bounds depend on the conditions $0 \leq(2 m-n+1)^{2}-4 n^{2}$, and $0 \leq \max _{x>1}(s(x))$ along with $x_{5}^{3} \leq\lfloor(2 m-n) / 2\rfloor$, respectively, which we will explain in detail.

The article is organized as follows. In $§ 2$ we give a short introduction to semi-regular sequences and Krawtchouk polynomials, and explain the connection between them. In $\S 3$ we relate the degree of regularity to the smallest root of Krawtchouk polynomials and translate information about the location of the smallest root to the degree of regularity. This involves an exact description of the degree of regularity as an eigenvalue problem as well as the translation of bounds. Since the eigenvalue problem seems to be intractable we focus on lower and upper bounds for the smallest root of Krawtchouk polynomials that are known to the literature, and derive the above claims in $\S 4, \S 5, \S 6, \S 7$. We conclude in $\S 8$ with concrete values and comparisons for illustration purposes.

\section{SEMI-REGULAR SEQUENCES AND KRAWTCHOUK POLYNOMIALS}

Let $f_{1}, \ldots, f_{m} \in \mathbf{K}\left[X_{1}, \ldots, X_{n}\right]$ be a system of polynomial equations where $\mathrm{K}$ is a field. We assume the system $f_{1}, \ldots, f_{m}$ to be zerodimensional, overdetermined and homogeneous quadratic, that is the graded commutative algebra $S=\mathrm{K}\left[X_{1}, \ldots, X_{n}\right] /\left(f_{1}, \ldots, f_{m}\right)$ is finite-dimensional, $m>n$ and the degree of each $f_{i}$ is 2 . We will adopt the usual notation for graded algebras and ideals, that is $S=\oplus_{j \geq 0} S_{j}$ and for an ideal $I<S$ generated by homogeneous elements $I=\oplus_{j \geq 0} I_{j}$.

Now, according to Bardet [3], Bardet et al. [5, 6], Diem [7] and Hodge et al. [12] such a system $f_{1}, \ldots, f_{m}$ of polynomial equations is defined to be a semi-regular sequence when the multiplication with any $f_{i}$ is injective in the graded algebra $S(i-1)=$ $\mathbf{K}\left[X_{1}, \ldots, X_{n}\right] /\left(f_{1}, \ldots, f_{i-1}\right)$ up to a certain degree. To be precise, $f_{1}, \ldots, f_{m}$ is semi-regular if the multiplication map

$$
\begin{aligned}
S(i-1)_{j} & \longrightarrow S(i-1)_{j+2} \\
g & \longmapsto g f_{i}
\end{aligned}
$$


is injective for each $i=1, \ldots, m$ and $j<d_{r e g}-2$ where $d_{r e g}$ is the degree of regularity of the graded ideal $J=\left(f_{1}, \ldots, f_{m}\right)$ given by

$$
d_{r e g}=\min \left\{d \geq 0: \operatorname{dim}_{\mathrm{K}} J_{d}=\operatorname{dim}_{\mathrm{K}} \mathrm{K}\left[X_{1}, \ldots, X_{n}\right]_{d}\right\} .
$$

By [6, Proposition 5 (i)] and [12, Theorem 2.3 (d)] the polynomial system $f_{1}, \ldots, f_{m}$ is semi-regular if and only if the Hilbert series of $S=\mathbf{K}\left[X_{1}, \ldots, X_{n}\right] /\left(f_{1}, \ldots, f_{m}\right)$ is

$$
\operatorname{HS}_{S}(z)=\left|\frac{\left(1-z^{2}\right)^{m}}{(1-z)^{n}}\right|_{+}=\left|(1-z)^{m-n}(1+z)^{m}\right|_{+} .
$$

Here, $\left|\sum_{k \geq 0} a_{k} z^{k}\right|_{+}$means truncation at the first non-positive coefficient. That is,

$$
\left|\sum_{k \geq 0} a_{k} z^{k}\right|_{+}=\sum_{\left\{k: \forall \forall_{l \leq k}\left(a_{l}>0\right)\right\}} a_{k} z^{k} .
$$

As noted in [6, Proposition 5 (iii)] the degree of regularity $d_{r e g}$ of a semi-regular sequence $f_{1}, \ldots, f_{m}$ is the index of the first nonpositive coefficient of $(1-z)^{m-n}(1+z)^{m}$, i.e.

$$
d_{r e g}\left(f_{1}, \ldots, f_{m}\right)=1+\operatorname{deg}\left(\operatorname{HS}_{S}(z)\right) \text {, }
$$

and consequently coincides with the Hilbert regularity of the graded algebra $S$. The degree of regularity is of great interest in the field of polynomial systems solving, since for semi-regular sequences the complexity of Faugère's F5 algorithm [9] for the computation of a Gröbner basis can be bounded by [6, Proposition 5 (iv)]

$$
O\left(m \cdot d_{r e g} \cdot\left(\begin{array}{c}
n+d_{r e g}-1 \\
d_{r e g}
\end{array}\right)^{\omega}\right)
$$

where $\omega<2.373$ is the exponent in the complexity of matrix multiplication. The expansion of the polynomial $(1-z)^{m-n}(1+z)^{m}$ allows the computation of the regularity for concrete instances when $m$ and $n$ are fixed. In particular, its $k$-th coefficient for $k=$ $0, \ldots, 2 m-n$ is

$$
\left[z^{k}\right](1-z)^{m-n}(1+z)^{m}=\sum_{j=0}^{k}(-1)^{j}\left(\begin{array}{c}
m-n \\
j
\end{array}\right)\left(\begin{array}{c}
m \\
k-j
\end{array}\right) .
$$

The alternating summation makes this explicit formula combinatorially unstable. That is, from this description it is virtually impossible to establish meaningful conditions on $k$ that imply $\left[z^{k}\right](1-z)^{m-n}(1+z)^{m}>0$.

An alternative approach to the coefficients is to understand the polynomial $(1-z)^{m-n}(1+z)^{m}$ as being the ordinary generating function of values of binary Krawtchouk polynomials at certain integers (see (4)). To recall those polynomials, we follow Levenshtein's exposition [19, (2)] (see also [15]) and denote by

$$
K_{k}^{N, r}(t)=\sum_{j=0}^{k}(-1)^{j}(r-1)^{k-j}\left(\begin{array}{l}
t \\
j
\end{array}\right)\left(\begin{array}{c}
N-t \\
k-j
\end{array}\right)
$$

the (general) Krawtchouk polynomial of degree $k$ for $k=0, \ldots, N$. From this one can deduce the ordinary generating function [19, (43)]:

$$
(w-z)^{x}(w+(r-1) z)^{N-x}=\sum_{k=0}^{N} K_{k}^{N, r}(x) \cdot z^{k} w^{N-k} .
$$

The Krawtchouk polynomials are discrete orthogonal polynomials associated to the binomial distribution via the orthogonality relation [19, Corollary 2.3]

$$
\sum_{i=0}^{N} K_{l}^{N, r}(i) K_{k}^{N, r}(i)(r-1)^{i}\left(\begin{array}{c}
N \\
i
\end{array}\right)=r^{N}(r-1)^{l}\left(\begin{array}{c}
N \\
l
\end{array}\right) \delta_{l, k}
$$

that holds for any $l, k=0, \ldots, N$. Here, $\delta_{l, k}$ denotes the Kronecker symbol. They can be computed from the recurrence relation [19, Corollary 3.3]

$$
\begin{array}{r}
(k+1) K_{k+1}^{N, r}(t)=(N(r-1)-k(r-2)-r t) K_{k}^{N, r}(t) \\
-(r-1)(N-k+1) K_{k-1}^{N, r}(t) .
\end{array}
$$

For our purposes we will only consider the binary Krawtchouk polynomials, that is $r=2$, and drop this parameter to simplify the notation. Then, the ordinary generating function (2) simplifies to

$$
(1-z)^{m-n}(1+z)^{m}=\sum_{k=0}^{2 m-n} K_{k}^{2 m-n}(m-n) \cdot z^{k} .
$$

Let us compute some binary Krawtchouk polynomials (Cf. Figure 1).

$$
\begin{aligned}
& K_{1}^{2 m-n}(t)= 2 m-n-2 t \\
& K_{2}^{2 m-n}(t)=\frac{1}{2}\left[\left(K_{1}^{2 m-n}(t)\right)^{2}-(2 m-n)\right] \\
& K_{3}^{2 m-n}(t)=\frac{1}{6}\left[\left(K_{1}^{2 m-n}(t)\right)^{3}-(3(2 m-n)-2)\left(K_{1}^{2 m-n}(t)\right)\right] \\
& K_{4}^{2 m-n}(t)=\frac{1}{24}\left[\left(K_{1}^{2 m-n}(t)\right)^{4}-(6(2 m-n)-8)\left(K_{1}^{2 m-n}(t)\right)^{2}\right. \\
&\quad+3(2 m-n-2)(2 m-n)]
\end{aligned}
$$

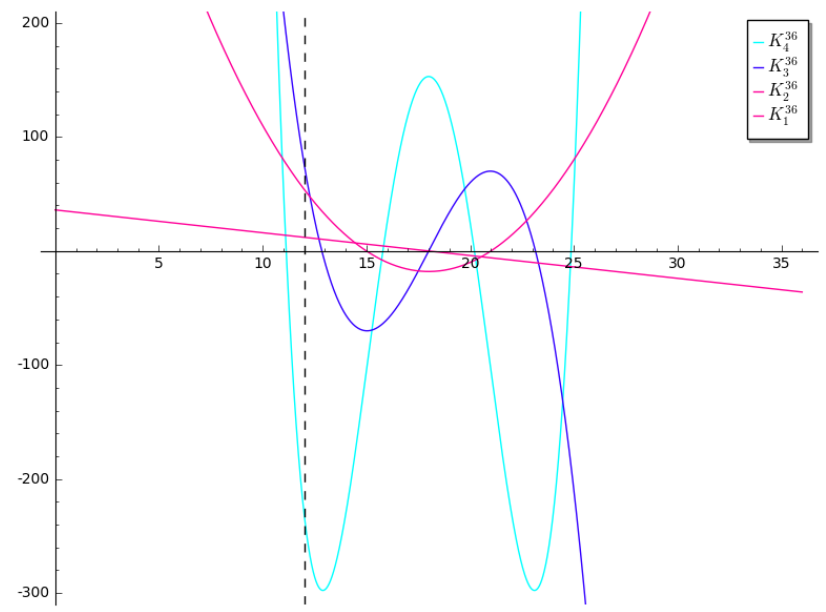

Figure 1: Some members of the family $K_{k}^{2 m-n}(t)$ for $m=24$ and $n=12$. The dashed line intersects the polynomials at their values at $t=12$, i.e. the first few coefficients of the generating function $(1-z)^{12}(1+z)^{24}=\sum_{k=0}^{36} K_{k}^{36}(12) \cdot z^{k}$. 
For further illustration we evaluate the above computed polynomials at $t=m-n$.

$$
\begin{aligned}
K_{1}^{2 m-n}(m-n) & =n \\
K_{2}^{2 m-n}(m-n) & =\frac{1}{2}\left[n^{2}+n-2 m\right] \\
K_{3}^{2 m-n}(m-n) & =\frac{1}{6}\left[n^{3}+3 n^{2}+2 n-6 m n\right] \\
K_{4}^{2 m-n}(m-n) & =\frac{1}{24}\left[n^{4}+6 n^{3}+(11-12 m) n^{2}+(6-12 m) n\right. \\
& \quad+12 m(m-1)]
\end{aligned}
$$

It is still challenging to unfold the recurrence relation (3) in order to predict $k$ such that $\left[z^{k}\right](1-z)^{m-n}(1+z)^{m}>0$. However, relation (4) allows a description of the degree of regularity (1) via roots of binary Krawtchouk polynomials as we will explain in §3.

\section{ROOTS OF KRAWTCHOUK POLYNOMIALS AND THE DEGREE OF REGULARITY}

We collect some properties of roots of orthogonal polynomials.

Theorem 3.1 (CF. [20, Theorem 3.3.1, Theorem 3.3.2]). Let $d_{k}^{N}(1), \ldots, d_{k}^{N}(k)$ denote the roots of the binary Krawtchouk polynomial $K_{k}^{N}$ where $k=1, \ldots, 2 m-n$. We have,

(1) the roots of $K_{k}^{N}$ are real, distinct and are located in the interior of the interval $[0, N]$, i.e. without loss of generality they are ordered as $0<d_{k}^{N}(1)<d_{k}^{N}(2)<\ldots<d_{k}^{N}(k)<N$.

(2) the roots of $K_{k}^{N}$ and $K_{k+1}^{N}$ interlace, i.e. for $k=1, \ldots, N-1$ and $j=1, \ldots, k$ we have $d_{k+1}^{N}(j)<d_{k}^{N}(j)<d_{k+1}^{N}(j+1)$.

The interlacing property allows to relate the degree of regularity of semi-regular sequences to the roots of binary Krawtchouk polynomials. In fact, this is the essential observation of this article.

Lemma 3.2. Let $f_{1}, \ldots, f_{m} \in \mathrm{K}\left[X_{1}, \ldots, X_{n}\right]$ be an overdetermined, zero-dimensional and homogeneous quadratic semi-regular sequence. The degree of regularity $d_{r e g}$ of $f_{1}, \ldots, f_{m}$ is given by

$$
d_{\text {reg }}=1+\max \left\{k: d_{k}^{2 m-n}(1)>m-n\right\},
$$

where $d_{k}^{2 m-n}(1)$ denotes the smallest root of $K_{k}^{2 m-n}$ for each $k=$ $1, \ldots, 2 m-n$.

Proof. Because of the interlacing property from Theorem 3.1 we have the following strictly decreasing sequence of smallest roots of the polynomials $K_{1}^{2 m-n}, \ldots, K_{2 m-n}^{2 m-n}$.

$$
d_{2 m-n}^{2 m-n}(1)<\ldots<d_{k+1}^{2 m-n}(1)<d_{k}^{2 m-n}(1)<\ldots<d_{1}^{2 m-n}(1)
$$

Hence, $d_{k}^{2 m-n}(1)>m-n$ implies $K_{l}^{2 m-n}(m-n)>0$ for all $l \leq k$. Conversely assume $K_{l}^{2 m-n}(m-n)>0$ for all $l \leq k$ and $d_{k}^{2 m-n}(1) \leq$ $m-n$. Since $K_{k}^{2 m-n}(0)=\left(\begin{array}{c}2 m-n \\ k\end{array}\right)>0$ and the roots are distinct, there must be an even number $e$ such that

$$
d_{k}^{2 m-n}(1)<\ldots<d_{k}^{2 m-n}(e)<m-n \leq d_{k}^{2 m-n}(e+1) .
$$

We choose a minimal such $k$ and note that $k>1$ since $K_{1}^{2 m-n}(t)=$ $2 m-n-2 t($ see $(5))$ and $d_{1}^{2 m-n}(1)=\frac{1}{2}(2 m-n)>m-n$. By the interlacing property each interval

$$
\left[d_{k}^{2 m-n}(1), d_{k}^{2 m-n}(2)\right], \ldots,\left[d_{k}^{2 m-n}(e-1), d_{k}^{2 m-n}(e)\right]
$$

contains exactly one root of $K_{k-1}^{2 m-n}$. Since $e$ is even, the number of those intervals is odd and since $K_{k-1}^{2 m-n}(0)=\left(\begin{array}{c}2 m-n \\ k-1\end{array}\right)>0$ we have either $K_{k-1}^{2 m-n}(m-n) \leq 0$ that contradicts the initial assumption, i.e. $K_{l}^{2 m-n}(m-n)>0$ for all $l \leq k$, or we have $d_{k}^{2 m-n}(e)<d_{k-1}^{2 m-n}(e)<m-n \leq d_{k}^{2 m-n}(e+1)$ which contradicts the minimality of $k$. Therefore,

$$
\left.\left\{k: \forall_{l \leq k}\left(K_{l}^{2 m-n}(m-n)>0\right)\right\}=\left\{k: d_{k}^{2 m-n}(1)>m-n\right)\right\},
$$

and for $S=\mathbf{K}\left[X_{1}, \ldots, X_{n}\right] /\left(f_{1}, \ldots, f_{m}\right)$ we have

$$
\begin{aligned}
\mathrm{HS}_{S}(z) & =\left|(1-z)^{m-n}(1+z)^{m}\right|_{+} K_{k}^{2 m-n}(m-n) \cdot z^{k} \\
& =\sum_{\left\{k: \forall_{l \leq k}\left(K_{l}^{2 m-n}(m-n)>0\right)\right\}} K_{k}^{2 m-n}(m-n) \cdot z^{k} . \\
& =\sum_{\left.\left\{k: d_{k}^{2 m-n}(1)>m-n\right)\right\}} \sum_{k} .
\end{aligned}
$$

In particular, $\operatorname{deg}\left(\operatorname{HS}_{S}(z)\right)=\max \left\{k: d_{k}^{2 m-n}(1)>m-n\right\}$.

By Lemma 3.2 it is clear, that any useful expression for the smallest roots of binary Krawtchouk polynomials yields a description of the degree of regularity of semi-regular sequences. Levenshtein [19] proves an expression based on the maximization of a quadratic form that we recollect.

Theorem 3.3 (CF. [19, Theorem 6.1]). Let $d_{k}^{2 m-n}(1)$ denote the smallest root of $K_{k}^{2 m-n}$ for each $k=1, \ldots, 2 m-n$. Then,

$$
d_{k}^{2 m-n}(1)=\frac{2 m-n}{2}-\max _{\|w\|_{2}^{2}=1}\left(\sum_{i=0}^{k-2} w_{i} w_{i+1} \sqrt{(i+1)(2 m-n-i)}\right) \text {. }
$$

This allows to describe the determination of the degree of regularity of a semi-regular sequence as an eigenvalue problem.

Lemma 3.4. Let $f_{1}, \ldots, f_{m} \in \mathbf{K}\left[X_{1}, \ldots, X_{n}\right]$ be as in Lemma 3.2. The degree of regularity $d_{r e g}$ of $f_{1}, \ldots, f_{m}$ is given by

$$
d_{\text {reg }}=1+\max \left\{k: \lambda_{k}^{2 m-n}<n\right\},
$$

where $\lambda_{k}^{2 m-n}$ denotes the largest eigenvalue of the real symmetric tridiagonal matrix $A_{k}^{2 m-n} \in \mathbf{R}^{k \times k}$ with non-zero entries only on the super- und subdiagonal as follows

$$
\begin{array}{rr}
\left(A_{k}^{2 m-n}\right)_{i j}=\sqrt{(i+1)(2 m-n-i)} & \text { for }|i-j|=1, \\
\left(A_{k}^{2 m-n}\right)_{i j}=0 & \text { otherwise, }
\end{array}
$$

with $i, j=0, \ldots, k-1$ and $k=1, \ldots, 2 m-n$.

Proof. This is a reformulation of Lemma 3.2 via Theorem 3.3 and standard linear algebra. That is,

$$
2 \cdot d_{k}^{2 m-n}(1)=2 m-n-2 \cdot \max _{\|w\|_{2}^{2}=1}\left(w^{t} \tilde{A} w\right)
$$

with $\tilde{A} \in \mathbf{R}^{k \times k}$ being non-zero on the superdiagonal as follows

$$
\begin{array}{lr}
(\tilde{A})_{i j}=\sqrt{(i+1)(2 m-n-i)} & \text { for } j-i=1, \\
(\tilde{A})_{i j}=0 & \text { otherwise, }
\end{array}
$$

with $i, j=0, \ldots, k-1$. We can replace $\tilde{A}$ by the symmetric matrix $\frac{1}{2}(\tilde{A}+\tilde{A})^{t}=\frac{1}{2} A_{k}^{2 m-n}$, where $A_{k}^{2 m-n}$ is given in the formulation of Lemma 3.2 above, without changing the quadratic form and obtain $2 \cdot d_{k}^{2 m-n}(1)=2 m-n-2 \cdot \max _{\|w\|_{2}^{2}=1}\left(w^{t} \frac{1}{2} A_{k}^{2 m-n} w\right)=2 m-n-\lambda_{k}^{2 m-n}$ 
where $\lambda_{k}^{2 m-n}$ denotes the largest eigenvalue of $A_{k}^{2 m-n}$. Consequently, by Lemma 3.2

$$
\begin{aligned}
d_{\text {reg }} & =1+\max \left\{k: d_{k}^{2 m-n}(1)>m-n\right\} \\
& =1+\max \left\{k: 2 m-n-\lambda_{k}^{2 m-n}>2(m-n)\right\} \\
& =1+\max \left\{k: \lambda_{k}^{2 m-n}<n\right\}
\end{aligned}
$$

The tridiagonal matrix of Lemma 3.4 is a Golub-Kahan matrix [10]. It appears that no explicit formulæ for the eigenvalues of such a matrix are known. Some general results on the explicit computation of eigenvalues of tridiagonal matrices are given by Kouachi [14]. Unfortunately those results do not apply to our matrix.

Instead of producing an exact expression for the degree of regularity of semi-regular sequences, our Lemma 3.2 allows us to immediately translate lower and upper bounds for the smallest root of binary Krawtchouk polynomials into bounds for the degree of regularity.

Lemma 3.5. Let $f_{1}, \ldots, f_{m} \in \mathbf{K}\left[X_{1}, \ldots, X_{n}\right]$ be as in Lemma 3.2 with degree of regularity $d_{\text {reg. }}$. Then,

$$
\begin{aligned}
& d_{\text {reg }} \geq 1+\max \left\{k: \operatorname{LB}_{k}^{2 m-n}(1)>m-n\right\}, \\
& d_{\text {reg }} \leq 1+\min \left\{k: \operatorname{UB}_{k}^{2 m-n}(1)<m-n\right\},
\end{aligned}
$$

where $\mathrm{LB}_{k}^{2 m-n}(1)$ and $\mathrm{UB}_{k}^{2 m-n}(1)$ are (not necessarily strict) lower and upper bounds, respectively, for the smallest root $d_{k}^{2 m-n}(1)$ of the binary Krawtchouk polynomial $K_{k}^{2 m-n}$ for each $k=1, \ldots, 2 m-n$. If the bounds $\mathrm{LB}_{k}^{2 m-n}(1)$ and $\mathrm{UB}_{k}^{2 m-n}(1)$ are indeed strict, then they are allowed to attain the threshold $m-n$, i.e.

$$
\begin{aligned}
& d_{r e g} \geq 1+\max \left\{k: \operatorname{LB}_{k}^{2 m-n}(1) \geq m-n\right\}, \\
& d_{r e g} \leq 1+\min \left\{k: \operatorname{UB}_{k}^{2 m-n}(1) \leq m-n\right\} .
\end{aligned}
$$

Proof. For the first part one has to realize that

$$
\left\{k: \operatorname{LB}_{k}^{2 m-n}(1)>m-n\right\} \subseteq\left\{k: d_{k}^{2 m-n}(1)>m-n\right\},
$$
and

$$
\begin{aligned}
& \left\{k: d_{k}^{2 m-n}(1)>m-n\right\} \\
& \quad \subseteq\left\{k: k \leq \min \left\{k^{\prime}: \mathrm{UB}_{k^{\prime}}^{2 m-n}(1)<m-n\right\}\right\} .
\end{aligned}
$$

The threshold assertions about strict bounds are obvious.

The following (strict) lower bounds on the smallest root of Krawtchouk polynomials have been reported in the literature.

Lemma 3.6 ([16, Corollary 1], [19, (125)], [20, (6.32.6)]). Consider the smallest root $d_{k}^{2 m-n}(1)$ of the binary Krawtchouk polynomial $K_{k}^{2 m-n}$. Then, for $1 \leq k<\frac{1}{2}(2 m-n)$ Krasikov and Zarkh [16, Corollary 1] give

$$
\begin{aligned}
& d_{k}^{2 m-n}(1)> \\
& \quad \frac{1}{2}(2 m-n)-\sqrt{k(2 m-n-k)}\left(1-\frac{3}{2}\left(\frac{2 m-n-2 k}{2 k(2 m-n-k)}\right)^{\frac{2}{3}}\right) .
\end{aligned}
$$

Furthermore, for each $k=1, \ldots, 2 m-n$ Levenshtein [19, (125)] in combination with an upper bound on the largest root $h_{k}$ of the Hermite polynomial $H_{k}(X)$ described by Szegó $[20,6.32 .6]$ gives

$$
\begin{aligned}
& d_{k}^{2 m-n}(1)> \\
& \quad \frac{1}{2}(2 m-n)-\sqrt{\frac{1}{2}(2 m-n)}\left(\sqrt{2 k+1}-6^{-\frac{1}{3}} i_{1}(2 k+1)^{-\frac{1}{6}}\right),
\end{aligned}
$$

where $i_{1}<i_{2}<i_{3}<\cdots$ are the real zeroes of the Airy's function $\mathcal{A}(x)$ that is a solution of the ordinary differential equation $y^{\prime \prime}+$ $\frac{1}{3} x y=0$ (see $\left.[20, \S 1.81]\right)$. Note that $i_{1} \approx 3.37213$ and $6^{-\frac{1}{3}} i_{1} \approx 1.85575$ [20, (6.32.7)].

We also consider the following (strict) upper bounds on the smallest root of Krawtchouk polynomials.

Lemma 3.7 ([18, (6.25)], [19, (124)], [20, (6.2.14)]). Consider the smallest root $d_{k}^{2 m-n}(1)$ of the binary Krawtchouk polynomial $K_{k}^{2 m-n}$. Then, for each $k=1, \ldots, 2 m-n$ Levenshtein [19, (124)] in combination with a lower bound on the largest root $h_{k}$ of the Hermite polynomial $H_{k}(X)$ described by Szegö [20, (6.2.14)] gives

$$
d_{k}^{2 m-n}(1)<\frac{1}{2}(2 m-n)-\frac{1}{2} \sqrt{(2 m-n-k+2)(k-1)} .
$$

Furthermore, for $1 \leq k \leq \frac{1}{2}(2 m-n)$ Levenshtein [18, (6.25)] (Cf. [15, (74)]) gives

$$
d_{k}^{2 m-n}(1)<\frac{1}{2}(2 m-n)-\left(k^{\frac{1}{2}}-k^{\frac{1}{6}}\right) \sqrt{2 m-n-k} .
$$

Figure 2 illustrates the lower, and Figure 3 additionally illustrates the upper bounds in a family of binary Krawtchouk polynomials. We will treat each of those bounds seperately to derive the corresponding bounds on the degree of regularity.

Note that there are further bounds present in the literature $[1,2$, 13] that apply to binary Krawtchouk polynomials. The results in [13, Theorem 3.2] give the upper bound $d_{k}^{2 m-n}(1)<\frac{1}{2}(2 m-n)$ and hence no extra information. The bounds established in [1, Corollary 5.2] coincide with (7). The bounds given in [1, Theorem 5.1 and Corollary 5.1] and [2, Theorem 1] will be subject to future research.

\section{LOWER BOUND ON THE REGULARITY FOLLOWING KRASIKOV AND ZARKH}

Theorem 4.1. Let $f_{1}, \ldots, f_{m} \in \mathbf{K}\left[X_{1}, \ldots, X_{n}\right]$ be as in Lemma 3.2. The smaller root of the polynomial $p(k)=k^{2}-(2 m-n) k+\frac{1}{4} n^{2}$ yields a lower bound for the degree of regularity as follows

$$
d_{\text {reg }} \geq 1+\left\lfloor\frac{1}{2}(2 m-n-2 \sqrt{m(m-n)})\right\rfloor .
$$

Proof. By Lemma 3.5 and (6) from Lemma 3.6 we have

$$
\begin{aligned}
d_{\text {reg }} \geq 1+\max \left\{k: m-n \leq \frac{1}{2}(2 m-n)\right. & \\
& \left.\quad-\sqrt{k(2 m-n-k)}\left(1-\frac{3}{2}\left(\frac{2 m-n-2 k}{2 k(2 m-n-k)}\right)^{\frac{2}{3}}\right)\right\},
\end{aligned}
$$

where the maximum is taken over $k=1, \ldots,\lfloor(2 m-n) / 2\rfloor$. Hence we seek the largest integer $1 \leq k \leq\lfloor(2 m-n) / 2\rfloor$ such that

$$
0 \leq \frac{n}{2}-\sqrt{k(2 m-n-k)}\left(1-\frac{3}{2}\left(\frac{2 m-n-2 k}{2 k(2 m-n-k)}\right)^{\frac{2}{3}}\right) .
$$




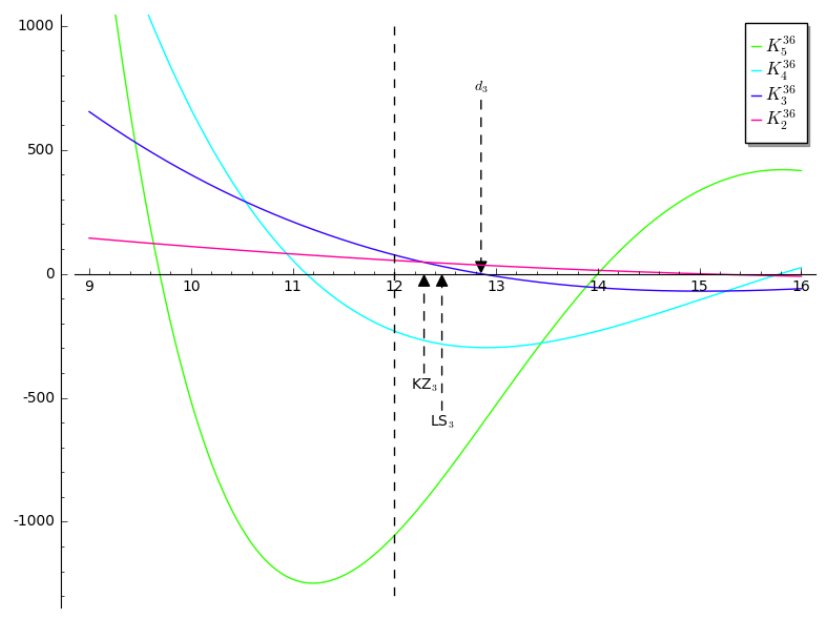

Figure 2: Members of the family $K_{k}^{36}$ associated to the generating function $(1-z)^{12}(1+z)^{24}=\sum_{k=0}^{36} K_{k}^{36}(12) \cdot z^{k}$. The plot shows that $K_{4}^{36}$ evaluates negative at 12 whereas $K_{3}^{36}(12)$ is positive. The first root of $K_{3}^{36}$ is $d_{3}^{36}(1) \approx 12.85$. The lower bound on $d_{3}^{36}(1)$ by Krasikov and Zarkh is $\mathrm{KZ}_{3} \approx 12.29$, Levenshtein and Szegő report $\mathrm{LS}_{3} \approx 12.47$.

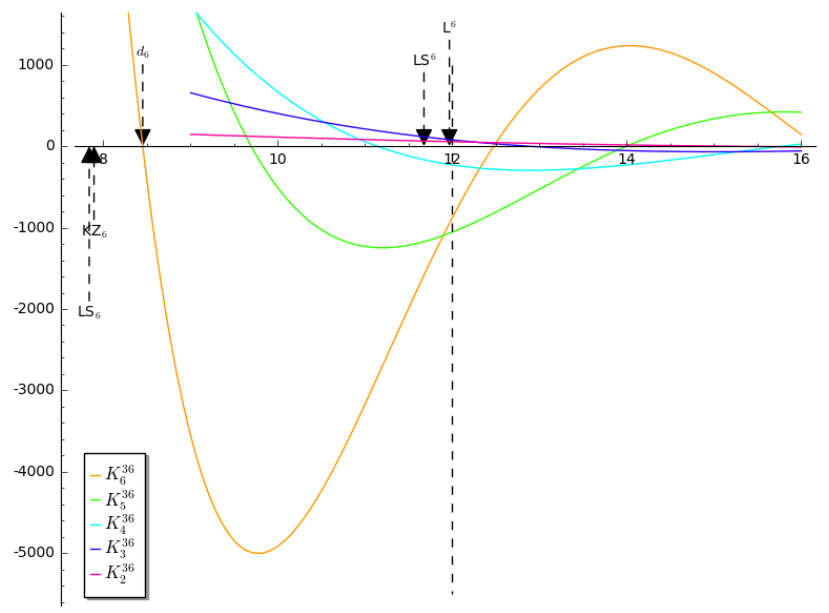

Figure 3: Members of the family $K_{k}^{36}$ associated to the generating function $(1-z)^{12}(1+z)^{24}=\sum_{k=0}^{36} K_{k}^{36}(12) \cdot z^{k}$. The plot shows that $K_{4}^{36}, K_{5}^{36}, K_{6}^{36}$ evaluate negative at 12 whereas $K_{3}^{36}(12)$ is positive. The first upper bounds in that family that are below 12 are those on $d_{6}^{36}(1)$, the first root of $K_{6}^{36}$. Now, $d_{6}^{36}(1) \approx 8.45$. Levenshtein and Szegő report $\mathrm{LS}^{6} \approx 11.68$, Levenshtein's upper bound is $L^{6} \approx 11.97$.

Now, for $1 \leq k \leq(2 m-n) / 2$ the term

$$
1-\frac{3}{2}\left(\frac{2 m-n-2 k}{2 k(2 m-n-k)}\right)^{\frac{2}{3}}
$$

is monotonically increasing, since its derivative (in $k$ ) is positive for any choice of $m>n$, and hence by simple evaluation at $k=1$ and $k=(2 m-n) / 2$ one concludes that it takes values in $(0,1]$. That is, we can simplify our consideration, seeking the largest integer $1 \leq k \leq\lfloor(2 m-n) / 2\rfloor$ such that

$$
0 \leq \frac{n}{2}-\sqrt{k(2 m-n-k)}
$$

since any such $k$ is valid also for (10) and hence gives a lower bound for the degree of regularity of $f_{1}, \ldots, f_{m}$. That is, we can equivalently consider the inequality

$$
0 \leq k^{2}-(2 m-n) k+\frac{1}{4} n^{2}
$$

The polynomial $p(k)=k^{2}-(2 m-n) k+\frac{1}{4} n^{2}$ has a positive discriminant $\operatorname{Disc}_{k}(p)=m(m-n)$, and hence real roots given by

$$
k_{1,2}=\frac{1}{2}(2 m-n \pm 2 \sqrt{m(m-n)}) .
$$

Moreover, since $k \leq\lfloor(2 m-n) / 2\rfloor$ we can identify our integer

$$
k \leq\left\lfloor\frac{1}{2}(2 m-n-2 \sqrt{m(m-n)})\right\rfloor .
$$

\section{LOWER BOUND ON THE REGULARITY FOLLOWING LEVENSHTEIN AND SZEGŐ}

Recall the real zero $i_{1} \approx 3.37213$ of the Airy's function $\mathcal{A}(x)$ described in Lemma 3.6.

Theorem 5.1. Let $f_{1}, \ldots, f_{m} \in \mathbf{K}\left[X_{1}, \ldots, X_{n}\right]$ be as in Lemma 3.2. The quartic polynomial

$$
q(w)=w^{4}-\frac{n}{\sqrt{2(2 m-n)}} w-6^{-\frac{1}{3}} i_{1}
$$

has a unique positive real root $w_{4}$, and the degree of regularity $d_{r e g}$ of $f_{1}, \ldots, f_{m}$ is bounded from below by

$$
d_{r e g} \geq 1+\left\lfloor\frac{1}{2}\left(w_{4}^{6}-1\right)\right\rfloor .
$$

Furthermore, with

$$
\begin{aligned}
& a=\frac{n}{\sqrt{2(2 m-n)}} \\
& b=-6^{-\frac{1}{3}} i_{1} \approx-1.85575
\end{aligned}
$$

we have

$$
w_{4}=\frac{1}{2}\left(\sqrt{U_{a, b}}+\sqrt{\frac{2 a}{\sqrt{U_{a, b}}}-U_{a, b}}\right) \text {, }
$$

where

$$
\begin{aligned}
& U_{a, b}=T_{a, b}^{\frac{1}{3}}-\frac{4 b}{3} T_{a, b}^{-\frac{1}{3}}, \\
& T_{a, b}=\frac{1}{2} a^{2}+\frac{1}{2} \sqrt{a^{4}+\frac{256}{27} b^{3}} .
\end{aligned}
$$

Proof. By Lemma 3.5 and (7) from Lemma 3.6 we have

$$
\begin{aligned}
d_{\text {reg }} \geq 1+\max & \left\{k: m-n \leq \frac{1}{2}(2 m-n)\right. \\
& \left.\quad-\sqrt{\frac{1}{2}(2 m-n)}\left(\sqrt{2 k+1}-6^{-\frac{1}{3}} i_{1}(2 k+1)^{-\frac{1}{6}}\right)\right\},
\end{aligned}
$$


where the maximum is taken over $k=1, \ldots, 2 m-n$. Hence we seek the largest integer $1 \leq k \leq 2 m-n$ such that

$$
0 \leq \frac{n}{2}-\sqrt{\frac{1}{2}(2 m-n)}\left(\sqrt{2 k+1}-6^{-\frac{1}{3}} i_{1}(2 k+1)^{-\frac{1}{6}}\right) .
$$

Since $m>n$ this is equivalent to consider

$$
0 \leq \frac{n}{\sqrt{2(2 m-n)}}-\sqrt{2 k+1}-6^{-\frac{1}{3}} i_{1}(2 k+1)^{-\frac{1}{6}} .
$$

We do a variable substitution

$$
k \mapsto \frac{1}{2}\left(w^{6}-1\right),
$$

and obtain the Laurent polynomial

$$
-w^{3}+6^{-\frac{1}{3}} i_{1} \frac{1}{w}+\frac{n}{\sqrt{2(2 m-n)}} .
$$

Note that we consider only $w \neq 0$ and that we have the rational function

$$
-\frac{1}{w}\left(w^{4}-\frac{n}{\sqrt{2(2 m-n)}} w-6^{-\frac{1}{3}} i_{1}\right) .
$$

So we are interested in the roots of the nominator which is the quartic polynomial given above

$$
q(w)=w^{4}-\frac{n}{\sqrt{2(2 m-n)}} w-6^{-\frac{1}{3}} i_{1} .
$$

Its discriminant $\operatorname{Disc}_{w}(q)$ is negative for any $m>n$ since

$$
\operatorname{Disc}_{w}(q)=-256\left(6^{-\frac{1}{3}} i_{1}\right)^{3}-27\left(\frac{n}{\sqrt{2(2 m-n)}}\right)^{4}<0 .
$$

Therefore $q$ has two complex conjugated roots $w_{1}, w_{2}$ and two real roots $w_{3} \leq w_{4}$. Moreover, since the constant term $-6^{-1 / 3} i_{1} \approx$ -1.85575 of the polynomial $q$ is negative there is a unique positive real root $w_{4}$ (Cf. Figure 4 ). Undoing the variable substitution (11) yields the claimed lower bound for the degree of regularity of $f_{1}, \ldots, f_{m}$. A symbolic computation in SageMath gives the expression for $w_{4}$ and finishes the proof.

Let us focus on the asymptotic growth of the lower bound given in Theorem 5.1. We adopt the usual notation of asymptotically equivalent functions, that is $f \sim g$ iff $\lim _{x \rightarrow \infty} f(x) / g(x)=1$.

Corollary 5.2. Assume $m$ grows subquadratic in $n$, i.e. $m=o\left(n^{2}\right)$. Then, as $n \rightarrow \infty$, the lower bound of Theorem 5.1 behaves as

$$
1+\left\lfloor\frac{1}{2}\left(w_{4}^{6}-1\right)\right\rfloor \sim \frac{n^{2}}{4(2 m-n)} .
$$

Proof. We borrow the notation from Theorem 5.1. For $m=$ $o\left(n^{2}\right)$ we have $a \rightarrow \infty, T_{a, b} \sim a^{2}$, and $U_{a, b} \sim a^{\frac{2}{3}}$. Hence,

$$
w_{4} \sim \frac{1}{2} \sqrt{a^{\frac{2}{3}}}+\frac{1}{2} \sqrt{\frac{2 a}{\sqrt{a^{\frac{2}{3}}}}-a^{\frac{2}{3}}}=\frac{1}{2} a^{\frac{1}{3}}+\frac{1}{2} \sqrt{2 a^{\frac{2}{3}}-a^{\frac{2}{3}}}=a^{\frac{1}{3}},
$$

and

$$
\frac{1}{2}\left(w_{4}^{6}-1\right) \sim \frac{1}{2}\left(a^{\frac{6}{3}}-1\right)=\frac{1}{2}\left(\frac{n^{2}}{2(2 m-n)}-1\right) \sim \frac{n^{2}}{4(2 m-n)} .
$$

We omit a deeper asymptotic analysis involving monotonicity considerations for reasons of brevity, but further summarize some interesting cases.

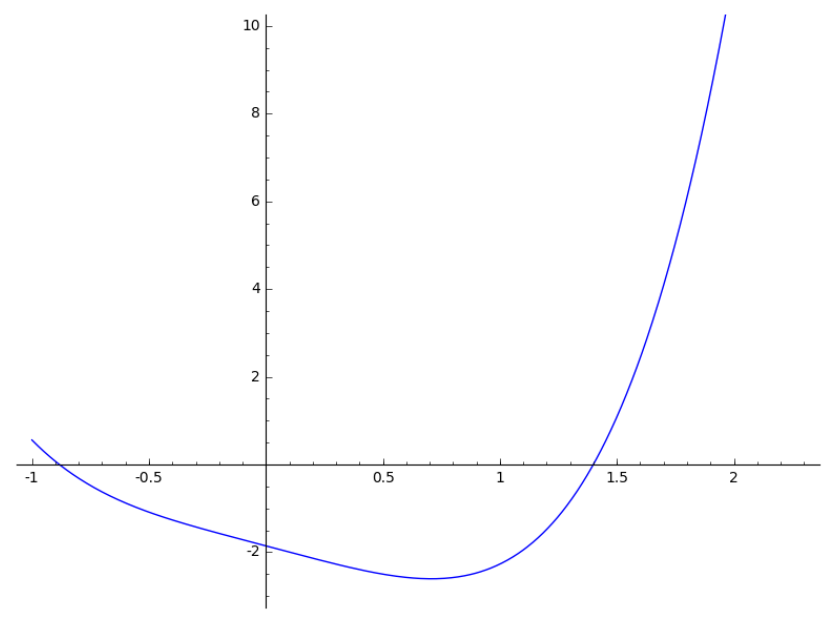

Figure 4: A plot of the quartic $w^{4}-n / \sqrt{2(2 m-n)} w-6^{-\frac{1}{3}} i_{1}$ for $m=24$ and $n=12$, i.e. of the polynomial $w^{4}-0.1179 w-$ 1.85575. The real roots are $w_{3} \approx-0.88$ and $w_{4} \approx 1.40$, hence $\frac{1}{2}\left(w_{4}^{6}-1\right) \approx 3.26$. Note the first non-positive coefficient in the expansion of $(1-z)^{12}(1+z)^{24}$ is the coefficient of $z^{4}$, and $d_{\text {reg }}=4 \geq 1+\lfloor 3.26\rfloor=4$.

Corollary 5.3. Let $\alpha, \beta>0$ and $\gamma \in(0,1)$ be real constants. Then, as $n \rightarrow \infty$, the lower bound of Theorem 5.1 behaves as

$$
1+\left\lfloor\frac{1}{2}\left(w_{4}^{6}-1\right)\right\rfloor \sim \begin{cases}\frac{n}{4\left(1+\frac{2 \alpha}{n}\right)} & , \text { for } m=n+\alpha, \\ \frac{n}{4(2 \beta-1)} & , \text { for } m=\beta n, \\ \frac{n}{4(2 \log (n)-1)} & , \text { for } m=n \log (n), \\ \frac{1}{8} n^{\gamma} & , \text { for } m=n^{2-\gamma} .\end{cases}
$$

Remark 5.4. Note that Corollary 5.3 carries similarities with the summary of Gröbner basis computation costs in $[4, \S 6]$, though the corresponding polynomial equations systems differ.

Remark 5.5. In the case when $m$ grows quadratically in $n$, i.e. $m=\delta n^{2}$ for some positive constant $\delta \in \mathbf{R}$, or when $m$ grows superquadratic in $n$, i.e. $m=\omega\left(n^{2}\right)$, the lower bound given in Theorem 5.1 tends to the value 2 . Those two cases behave as expected. As the number of quadratic semi-regular (i.e. in this sense algebraically indepent) equations becomes large, the Macaulay matrix already contains all homogeneous entries of degree 2 whose total number is $\left(\begin{array}{c}n+2+1 \\ n+1\end{array}\right) \sim \frac{1}{2} n^{2}$.

\section{UPPER BOUND ON THE REGULARITY FOLLOWING LEVENSHTEIN AND SZEGŐ}

THeorem 6.1. Let $f_{1}, \ldots, f_{m} \in \mathbf{K}\left[X_{1}, \ldots, X_{n}\right]$ be as in Lemma 3.2. If the discriminant $\operatorname{Disc}_{k}(t)=(2 m-n+1)^{2}-4 n^{2}$ of the polynomial $t(k)=k^{2}-(2 m-n+3) k+2 m-n+2+n^{2}$ is non-negative, then its smaller root yields a lower bound for the degree of regularity as follows

$$
d_{r e g} \leq 1+\left\lceil\frac{1}{2}\left(2 m-n+3-\sqrt{(2 m-n+1)^{2}-4 n^{2}}\right)\right\rceil .
$$


Proof. By Lemma 3.5 and (8) from Lemma 3.7 we have

$$
\begin{aligned}
& d_{r e g} \leq 1+\min \left\{k: m-n \geq \frac{1}{2}(2 m-n)\right. \\
&\left.-\frac{1}{2} \sqrt{(2 m-n-k+2)(k-1)}\right\},
\end{aligned}
$$

where the minimum is taken over $k=1, \ldots, 2 m-n$. Hence we seek the smallest integer $1 \leq k \leq 2 m-n$ such that

$$
n \leq \sqrt{(2 m-n-k+2)(k-1)} .
$$

We square (12) and obtain the inequality

$$
0 \geq k^{2}-(2 m-n+3) k+\left(2 m-n+2+n^{2}\right) .
$$

The roots of the quadratic polynomial

are

$$
t(k)=k^{2}-(2 m-n+3) k+\left(2 m-n+2+n^{2}\right)
$$

$$
k_{1,2}=\frac{1}{2}\left(2 m-n+3 \pm \sqrt{(2 m-n+1)^{2}-4 n^{2}}\right) .
$$

They are real in the case of a non-negative discrimant, i.e.

$$
0 \leq \operatorname{Disc}_{k}(t)=(2 m-n+1)^{2}-4 n^{2} .
$$

Recall that we are interested in the smallest integer $k \leq 2 m-n$ that satisfies (12). Hence, under the non-negativity condition (13) we have

$$
d_{\text {reg }} \leq 1+\left\lceil\frac{1}{2}\left(2 m-n+3-\sqrt{(2 m-n+1)^{2}-4 n^{2}}\right)\right\rceil .
$$

Remark 6.2. In contrast to the lower bounds established in Theorem 4.1 and Theorem 5.1, which exist for any $m>n$, the upper bound in Theorem 6.1 depends on a non-negative discriminant $\operatorname{Disc}_{k}(t)=(2 m-n+1)^{2}-4 n^{2}$. This can be interpreted in terms of the family of Krawtchouk polynomials. Our Figure 3 actually illustrates the non-negative case. In the case of a negative discriminant, the set $\left\{k: \mathrm{UB}_{k}^{2 m-n}(1) \leq m-n\right\}$ from Lemma 3.5, with $\mathrm{UB}_{k}^{2 m-n}(1)$ being the upper bound of Levenshtein and Szegő (see (8) in Lemma 3.7), is empty. That is, for any family member this upper bound does not pass $m-n$.

\section{UPPER BOUND ON THE REGULARITY FOLLOWING LEVENSHTEIN}

Theorem 7.1. Let $f_{1}, \ldots, f_{m} \in \mathbf{K}\left[X_{1}, \ldots, X_{n}\right]$ be as in Lemma 3.2. The sextic polynomial

$$
s(x)=x(x-1)^{2}\left(2 m-n-x^{3}\right)-\frac{1}{4} n^{2}
$$

has a global maximum at some $x^{\prime} \in\left(1,(2 m-n)^{1 / 3}\right)$. If $s\left(x^{\prime}\right) \geq 0$, then $s$ has a a unique real root $x_{5} \in\left(1, x^{\prime}\right]$. If $x_{5}^{3} \leq\lfloor(2 m-n) / 2\rfloor$, then

$$
d_{\text {reg }} \leq 1+\left\lceil x_{5}^{3}\right\rceil \text {. }
$$

Proof. By Lemma 3.5 and (9) from Lemma 3.7 we have

$d_{r e g} \leq 1+\min \left\{k: m-n \geq \frac{2 m-n}{2}-\left(k^{\frac{1}{2}}-k^{\frac{1}{6}}\right) \sqrt{2 m-n-k}\right\}$, where the minimum is taken over $k=1, \ldots,\lfloor(2 m-n) / 2\rfloor$. Hence we seek the smallest integer $1 \leq k \leq\lfloor(2 m-n) / 2\rfloor$ such that

$$
\frac{n}{2} \leq\left(k^{\frac{1}{2}}-k^{\frac{1}{6}}\right) \sqrt{2 m-n-k}=\left(k^{\frac{1}{3}}-1\right) \sqrt{k^{\frac{1}{3}}(2 m-n-k)} .
$$

We do a variable substitution $k \rightarrow x^{3}$ and square (14) to obtain

$$
\frac{1}{4} n^{2} \leq x(x-1)^{2}\left(2 m-n-x^{3}\right) .
$$

Therefore we are interested in the roots of the sextic equation

$$
s(x)=x(x-1)^{2}\left(2 m-n-x^{3}\right)-\frac{1}{4} n^{2} .
$$

The sextic $s$ has a local extremum at 1 and by Rolle's lemma local extrema inside $(0,1)$ and $\left(1,(2 m-n)^{1 / 3}\right)$ (Cf. Figure 5). We look at the derivative of $s$, that is

$$
s^{\prime}(x)=(1-x)\left(6 x^{4}-4 x^{3}-3 x(2 m-n)+(2 m-n)\right),
$$

The discriminant of the quartic factor $r$ of the derivative $s^{\prime}$ is

$$
\operatorname{Disc}_{x}(r)=-78732(2 m-n)^{4}-39744(2 m-n)^{3}-6912(2 m-n)^{2}
$$

and hence negative for $m>n$. Therefore $r$ has two complex conjugate roots and two real roots $x_{3}^{\prime}<x_{4}^{\prime}$. This shows that the sextic has exactly three local extrema at $1, x_{3}^{\prime} \in(0,1)$ and $x_{4}^{\prime} \in\left(1,(2 m-n)^{1 / 3}\right)$. A second derivative test with some further computations show that $s$ has a local minimum at 1 and local maxima at $x_{3}^{\prime}, x_{4}^{\prime}$ if $m>n$. We now focus on the interval $\left(1,(2 m-n)^{1 / 3}\right)$ since the initial assumption $1 \leq k \leq\lfloor(2 m-n) / 2\rfloor$ and variable substitution $k=x^{3}$ puts the restriction $x \in\left[1,\lfloor(2 m-n) / 2\rfloor^{1 / 3}\right]$ on those $x$ that we consider valid to satisfy (15). Note that $s(1)=-n^{2} / 4<0$. That is, if $s\left(x_{4}^{\prime}\right) \geq 0$, then by the intermediate value theorem we have a unique real root $x_{5} \in\left(1, x_{4}^{\prime}\right]$ that satisfies (15). After undoing the variable substitution our $k=x_{5}^{3}$ satisfies (14) if $x_{5}^{3}$ is in the valid range, i.e. $x_{5}^{3} \leq\lfloor(2 m-n) / 2\rfloor$, and consequently $d_{\text {reg }} \leq 1+\left\lceil x_{5}^{3}\right\rceil$.

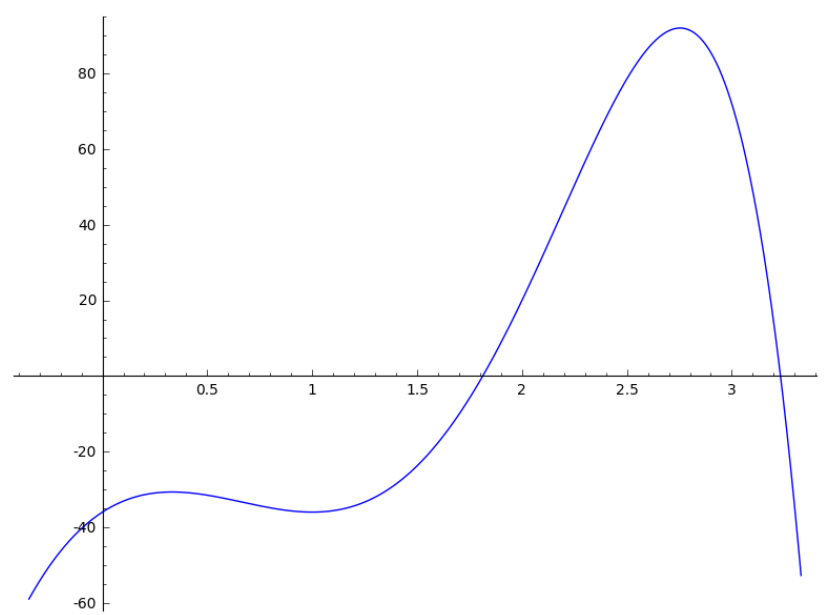

Figure 5: A plot of the sextic $x(x-1)^{2}\left(2 m-n-x^{3}\right)-n^{2} / 4$ for $m=24$ and $n=12$. The real roots are $x_{5} \approx 1.81$ and $x_{6} \approx$ 3.23. Now, the root in question is $x_{5}$ and undoing the variable substitution yields the upper bound $1+\left\lceil x_{5}^{3}\right\rceil=7$. The first non-positive coefficient in the expansion of $(1-z)^{12}(1+z)^{24}$ is the coefficient of $z^{4}$, and $d_{r e g}=4 \leq 7$. 
Remark 7.2. The sextic of Theorem 7.1 turns out to be irreducible with full Galois group $\Xi_{6}$ for almost all combinations $m>n$. Hence the methods of Hagedorn [11] for solving a solvable sextic are not applicable. For almost all remaining combinations $m>n$ it factors into a linear and quintic polynomial with full Galois group $\Xi_{5}$ Again, methods for solving a solvable quintic [8] do not apply. But in some of those cases the linear factor coincides with the root that gives our upper bound. For concrete instances though, the root $x_{5}$ can be determined by a numerical approximation via a root-finding algorithm.

Remark 7.3. The conditions for the existence of the upper bound in Theorem 7.1, i.e. $0 \leq \max _{x>1}(s(x))$ and $x_{5}^{3} \leq\lfloor(2 m-n) / 2\rfloor$, can be interpreted in complete analogy to Remark 6.2.

Remark 7.4. The position of the local maximum $x^{\prime}$ of the sextic $s$ in Theorem 7.1 can be given explicitely by a symbolic computation in SageMath applied to the quartic factor in (16).

\section{CONCRETE VALUES AND COMPARISONS}

The following is a collection of tables illustrating the lower bounds $\mathrm{KZ}_{\geq}, \mathrm{LS}_{\geq}$from Theorem 4.1 and Theorem 5.1, and the upper bounds $\mathrm{LS}^{\leq}, \mathrm{L} \leq$ from Theorem 6.1 and Theorem 7.1, respectively. They are put in contrast to the asymptotic estimates of Bardet et al. [6, Theorem 1], where we simply omitted the asymptotic term. Note that the Airy function considered in $[6,(3)]$ which is a solution of the differential equation $y^{\prime \prime}-x y=0$ is not the Airy function considered here in (7) from Lemma 3.6 and Theorem 5.1.

\begin{tabular}{|c|c|c|c|c|c|c|}
\hline \multicolumn{7}{|c|}{$m=n+100$} \\
\hline$n$ & $d_{\text {reg }}$ & {$[6,(2)]$} & $\mathrm{KZ}_{\geq}$ & $\mathrm{LS}_{\geq}$ & $\mathrm{LS}^{\leq}$ & $\mathrm{L}^{\leq}$ \\
\hline 256 & 48 & -0.86 & 40 & 44 & - & 75 \\
\hline 512 & 121 & 71.48 & 109 & 103 & - & 184 \\
\hline 1024 & 294 & 244.18 & 277 & 228 & - & 448 \\
\hline 2048 & 684 & 634.64 & 661 & 485 & - & - \\
\hline 4096 & 1534 & 1483.93 & 1501 & 1000 & - & - \\
\hline 8192 & 3333 & 3282.76 & 3286 & 2029 & - & - \\
\hline 16384 & 7075 & 7024.89 & 7009 & 4084 & - & - \\
\hline 32768 & 14766 & 14715.35 & 14672 & 8189 & - & - \\
\hline \multicolumn{7}{|c|}{$m=n+256$} \\
\hline$n$ & $d_{\text {reg }}$ & {$[6,(2)]$} & $\mathrm{KZ}_{\geq}$ & $\mathrm{LS}_{\geq}$ & $\mathrm{LS} \leq$ & $\mathrm{L}^{\leq}$ \\
\hline 256 & 29 & -95.87 & 22 & 28 & 100 & 46 \\
\hline 512 & 79 & -46.95 & 69 & 73 & 492 & 116 \\
\hline 1024 & 210 & 83.65 & 196 & 184 & - & 294 \\
\hline 2048 & 532 & 405.58 & 513 & 427 & - & 724 \\
\hline 4096 & 1277 & 1150.14 & 1249 & 933 & - & 1741 \\
\hline 8192 & 2977 & 2794.71 & 2882 & 1957 & - & - \\
\hline 16384 & 6442 & 6314.05 & 6385 & 4009 & - & - \\
\hline 32768 & 13814 & 13686.09 & 13733 & 8113 & - & - \\
\hline
\end{tabular}

\begin{tabular}{|c|c|c|c|c|c|c|}
\hline \multicolumn{7}{|c|}{$m=2 n$} \\
\hline$n$ & $d_{\text {reg }}$ & {$[6,(3)]$} & $\mathrm{KZ}_{\geq}$ & $\mathrm{LS}_{\geq}$ & $\mathrm{LS}^{\leq}$ & $\mathrm{L}^{\leq}$ \\
\hline 256 & 29 & 27.10 & 22 & 28 & 100 & 46 \\
\hline 512 & 52 & 50.79 & 44 & 51 & 198 & 78 \\
\hline 1024 & 98 & 96.87 & 88 & 96 & 393 & 139 \\
\hline 2048 & 189 & 187.45 & 176 & 184 & 785 & 253 \\
\hline 4096 & 368 & 366.58 & 352 & 358 & 1567 & 469 \\
\hline 8192 & 724 & 722.29 & 703 & 703 & 3131 & 884 \\
\hline 16384 & 1432 & 1430.51 & 1406 & 1391 & 6260 & 1687 \\
\hline 32768 & 2844 & 2842.91 & 2812 & 2763 & 12519 & 3249 \\
\hline
\end{tabular}

\begin{tabular}{|c|c|c|c|c|c|c|}
\hline \multicolumn{7}{|c|}{$m=8 n$} \\
\hline$n$ & $d_{\text {reg }}$ & {$[6,(3)]$} & $\mathrm{KZ}_{\geq}$ & $\mathrm{LS}_{\geq}$ & $\mathrm{LS}^{\leq}$ & $\mathrm{L}^{\leq}$ \\
\hline 256 & 8 & 6.57 & 5 & 8 & 20 & 14 \\
\hline 512 & 14 & 11.83 & 9 & 14 & 37 & 23 \\
\hline 1024 & 23 & 21.61 & 18 & 23 & 71 & 37 \\
\hline 2048 & 42 & 40.26 & 35 & 42 & 140 & 63 \\
\hline 4096 & 78 & 76.41 & 69 & 78 & 277 & 111 \\
\hline 8192 & 149 & 147.23 & 137 & 149 & 551 & 201 \\
\hline 16384 & 289 & 287.05 & 274 & 288 & 1100 & 371 \\
\hline 32768 & 566 & 564.37 & 547 & 565 & 2197 & 696 \\
\hline
\end{tabular}

\begin{tabular}{|l|rrrrrr|}
\hline \multicolumn{7}{|l|}{$m=n \log _{2}(n)$} \\
\hline$n$ & $d_{\text {reg }}$ & {$[6]$} & $\mathrm{KZ}_{\geq}$ & $\mathrm{LS}_{\geq}$ & $\mathrm{LS} \leq$ & $\mathrm{L} \leq$ \\
\hline 256 & 8 & - & 5 & 8 & 20 & 14 \\
512 & 12 & - & 8 & 12 & 33 & 21 \\
1024 & 19 & - & 14 & 19 & 57 & 31 \\
2048 & 31 & - & 25 & 31 & 100 & 48 \\
4096 & 53 & - & 45 & 53 & 181 & 78 \\
8192 & 92 & - & 82 & 92 & 331 & 129 \\
16384 & 164 & - & 152 & 164 & 610 & 220 \\
32768 & 298 & - & 283 & 298 & 1134 & 382 \\
\hline
\end{tabular}

\section{ACKNOWLEDGEMENTS}

I would like to thank Max Gebhardt, Jernej Tonejc and Andreas Wiemers for helpful discussions.

\section{REFERENCES}

[1] Iván Area, Dimitar K. Dimitrov, Eduardo Godoy, and Vanessa Paschoa. 2013. Zeros of classical orthogonal polynomials of a discrete variable. Math Comp. 82 (2013), 1069-1095.

[2] Iván Area, Dimitar K. Dimitrov, Eduardo Godoy, and Vanessa Paschoa. 2015. Bounds for the zeros of symmetric Kravchuk polynomials. Numerical Algorithms 69, 3 (2015), 611-624.

[3] Magali Bardet. 2004. Étude des systèmes algébriques surdéterminés. Applications aux codes correcteurs et à la cryptographie. Ph.D. Dissertation. Université Pierre et Marie Curie - Paris VI.

[4] Magali Bardet, Jean-Charles Faugère, and Bruno Salvy. 2003. Semi-regular overdetermined sequences over $\mathbf{F}_{2}$ with solutions in $\mathbf{F}_{2}$. INRIA, Research Report (2003).

[5] Magali Bardet, Jean-Charles Faugère, and Bruno Salvy. 2004. On the complexity of Gröbner basis computation of semi-regular overdetermined algebraic equations. In Proceedings of ICPSS 2004, International Conference on Polynomial System Solving.

[6] Magali Bardet, Jean-Charles Faugère, Bruno Salvy, and Bo-Yin Yang. 2005. Asymptotic behaviour of the degree of regularity of semi-regular polynomial systems. In Proceedings of MEGA 2005, The Eighth International Symposium on Effective Methods in Algebraic Geometry.

[7] Claus Diem. 2015. Bounded regularity. Journal of Algebra 423 (2015), 1143-1160.

[8] David S. Dummit. 1991. Solving solvable quintics. Mathematics of Computation 57, 195 (1991), 387-401.

[9] Jean Charles Faugère. 2002. A new efficient algorithm for computing Gröbner bases without reduction to zero (F5). In Proceedings of the 2002 International Symposium on Symbolic and Algebraic Computation (ISSAC '02). 75-83. 
[10] Gene Golub and William Kahan. 1965. Calculating the singular values and pseudoinverse of a matrix. Fournal of the Society for Industrial and Applied Mathematics, Series B: Numerical Analysis 2, 2 (1965), 205-224.

[11] Thomas R. Hagedorn. 2000. General formulas for solving solvable sextic equations. Fournal of Algebra 233, 2 (2000), 704-757.

[12] Timothy J. Hodges, Sergio Molina, and Jacob Schlather. 2017. On the existence of homogeneous semi-regular sequences in $\mathrm{F}_{2}\left[X_{1}, \ldots, X_{n}\right] /\left(X_{1}^{2}, \ldots, X_{n}^{2}\right)$. Journal of Algebra 476 (2017), 519-547.

[13] Alta Jooste and Kerstin Jordaan. 2014. Bounds for zeros of Meixner and Kravchuk polynomials. LMS fournal of Computation and Mathematics 17, 1 (2014), 47-57.

[14] Said Kouachi. 2006. Eigenvalues and eigenvectors of tridiagonal matrices. ELA. The Electronic Journal of Linear Algebra 15 (2006).

[15] Ilia Krasikov and Simon Litsyn. 2001. Survey of binary Krawtchouk polynomials. In Proceedings of Codes and Association Schemes 1999. DIMACS Series in Discrete
Mathematics and Theoretical Computer Science, Vol. 56.

[16] Ilia Krasikov and Alexander Zarkh. 2009. On zeros of discrete orthogonal polynomials. Journal of Approximation Theory 156, 2 (2009), 121-141.

[17] Mikhailo Krawtchouk. 1929. Sur une généralisation des polynômes d'Hermite. Comptes Rendus Hebdomadaires des Séances de l'Académie des Sciences, Paris 189 (1929), 620-622.

[18] Vladimir Levenshtein. 1983. Bounds for packings of metric spaces and some their applications. Problemi Kybernetiki 40 (1983), 43-110.

[19] Vladimir Levenshtein. 1995. Krawtchouk polynomials and universal bounds for codes and designs in Hamming spaces. IEEE Transactions on Information Theory 41, 5 (1995), 1303-1321.

[20] Gábor Szegő. 1975. Orthogonal polynomials (4 ed.). Number 23 in American Mathematical Society colloquium publications. 